
DOE/ER/54131--1

DE92 011806

\title{
NON-GEOMETRICAL OPTICS STUDY OF WAVE PROPAGATION
}

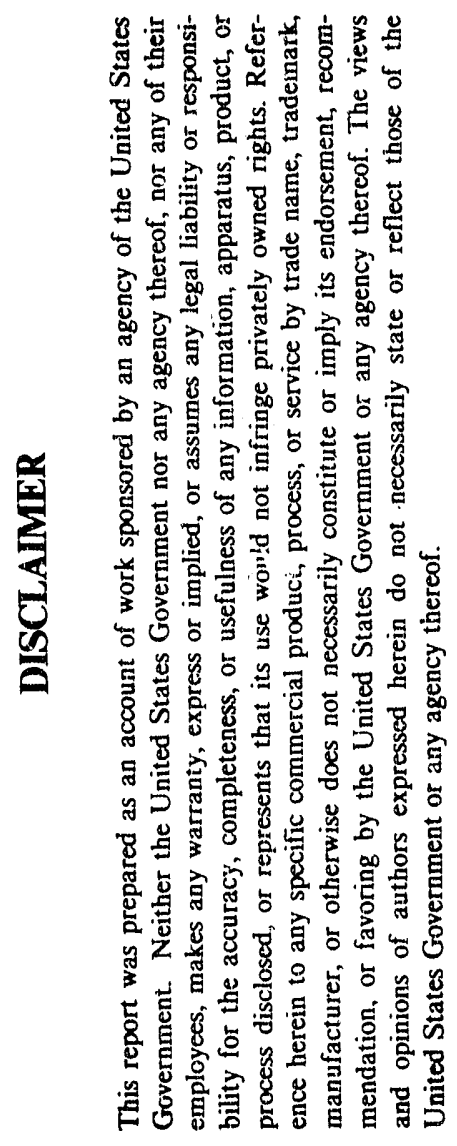

\author{
Annual Progress Report \\ for the period August 1991 to March 1992 \\ Kaya Imre \\ City University of New York \\ College of Staten Island \\ March 1992
}

Prepared for

THE U. S. DEPARTMENT OF ENERGY

GRANT NO. DE-FG02-91 ER 54131

MASTER 


\begin{abstract}
ABSTRAC'T
A statistical theory of growth curve analysis, which makes use of smoothing splines is formulated and coded for applications. We have tested our codes extensively using computer generated data superposed with random noise of various intensities, by simultaneously fitting all data with two independent variables. We are presently evaluating various experimental data in the MFE database by making use of our codes. Our preliminary results indicate that TFTR ohmic temperature profile shape depends almost exclusively on the edge plasma safety factor, $q_{a}$, as was previously shown to be true for ASDEX plasma profiles.

The main advantage of the utilization of a simultaneous fitting of all (appropriately normalized) profiles as a function of more than one plasma parameter, is to improve significantly the signal to noise ratio. We also determine the amount of smoothing to be applied during the curve fitting process. An optimally selected smoothing helps to reduce the random noise, but yet allows the predictions to stay close to the data. In addition, the use of a multi-dimensional parameter field provides us with a uniformly valid representation.

The curve fitting technique we employ uses a piecewise polynomial spline representation. However, we have eliminated the usually adopted method of setting a simple knot at every data point, for the reason that the experimientally obtained data is usually confined to the values of the plasma parameters bunched together in small intervals which are widely separated. Presently, we are using equally spaced knots.
\end{abstract}




\section{INTRODUCTION}

An essential part of the fusion research is to analyze and evaluate in dept the experimental data obtained from various fusion devices. Only by the application of advanced statistical techniques to these data can it become possible to bring out the hidden fine structures of the properties of the measured plasma variables, and in turn significantly improve our understanding of the physical phenomena associated with these experiments.

The use of smoothing splines in studying noisy data has been applied to various problems in the past. In most of these studies a scheme similar to that defined below is used. Given the approximate values $y_{i}=g\left(x_{i}\right)+\varepsilon_{i}$, of some smooth function $g$ at the data points $x_{i}, i=1, \ldots, N$, and an estimate $\sigma_{i}$ of standard deviation in $y_{i}$ one minimizes the functional

$$
\sum_{i=1}^{N}\left\{\frac{y_{i}-f\left(x_{i}\right)}{\sigma_{i}}\right\}^{2}+\lambda \int\left|f^{(\gamma)}(t)\right|^{m} d t
$$

over all functions with $\gamma$ derivatives. The parameter $\lambda$ controls the amount of smoothing to be applied on the given noisy data. Excessive smoothing of the data causes the predicted values to move away from the given data points. Thus, one has to use some criteria in order to decide the amount of smoothing and yet stay reasonably close to the data.

It is well-known that the solution of this problem can be expressed in terms of a piecewise polynomial spline representation with simple knots at the data points. We find that this selection is not quite suitable for data which has large numbers of data points in small intervals which are widely separated, as is the case for the plasma variables as functions of certain plasma parameters. In such cases the approximating function and its derivatives may develop a spurious oscillatory behavior which forces us to use a higher degree of smoothing. We have developed ccdes which not only let us place the knots appropriately, but also allow us to study the data $\varepsilon$. a function of more than one plasma parameter. In this way we can increase the number of input data points fed into the curve fitting-smoothing scheme, and thus we can both reduce noise level by a factor of $\sqrt{\mathrm{N}}$, and also obtain a uniformly valid represention. In Figure 1 we give an example of such data constructed by adding a random noise to a double Gaussian distribution which evolves with a second parameter $t$. The result of the smoothing spline code is shown in Figure 2.

We have also performed a preliminary study on the TFTR data obtained from the MFE profile database. We studied th: electron temperature $T_{e}$ as a function of normalized flux radius $\rho=r / a$ 
and the safety factor $q$, for the data obtained from 80 distinct ohmic discharge shots. In order to obtain a uniform input set, we have normalized the data for each shot as

$$
\begin{gathered}
\log T_{e}\left(\rho, q_{i}\right)=\beta_{i}+f_{i}(\rho)+g_{i}(\rho) \log q_{i}, \\
\beta_{i}=(1 / N) \Sigma_{k=1, \ldots, N} \log T_{e}\left(\rho_{k}, q_{i}\right) .
\end{gathered}
$$

The results of our preliminary study of the electron temperature are given in Figure 3. The normalized input data is shown for some selected values of $q$ in solid lines, and the smoothed spline fitting which is obtained from our code corresponding to this data is plotted in dashed lines, as a function of the flux radius. The three dimensional smoothed $T_{e}$ surface is presented in Figure 4. The result given in Figures 3 and 4, confirms the findings of Refs.1 and 3. Namely, we find that the ohmic temperature profile shape depends almost exclusively on the edge plasma $q$.

In summary, the following advantages result from a statistical parametrization of the plasma profiles. First, by simultaneously fitting all profiles with spline coefficients which depend on the plasma variables, such as $q_{a}$, a uniformly valid representation is obtained. Second, the signal to noise ratio is significantly improved, thus enabling us to obtain more reliable estimates for the functions and their derivatives.

A parametrized representation provides a compact summary of an experimental investigation: a table of spline coefficients is much more usable than a large database of all discharges. Knowledge of the dependence of profiles on the plasma parameters may lead to new physical insight. In view of the considerable experimental variation in individual profile measurements, it is essential to express clearly at least the statistical uncertainties associated with fitting a set of carefully measured profiles. Such parametrized profiles are then in a form suitable to be input into transport and stability simulations.

\section{Results Obtained}

We have demonstrated the feasiblity of the growth curve analysis based on a piecewise polynomial smoothing spline scheme in evaluating the experimental data. Presently we are continuing our studies by trying to improve further our method with the inclusion of some appropriate criteria for the degree of smoothing, so that the noise level in the raw data can be reduced, while retaining the accuracy of our estimates of the measured values. 
The principal investigator has devoted his two-thirds of his time to the present DOE account Grant No. DE-FG02-91-ER54131 since August 1991, with one half of this time paid by the College of Staten Island, City University of New York.

\section{REFERENCES}

1. P.J. McCarthy, K.S. Riedel, O.J.W.F. Kardaun, H. Murmann, K. Lackner, the ASDEX Team "Scaling and Plasma Profile Parameterization of ASDEX High Density Ohmic Discharges" Max-Planck Institut fur Plasmaphysik, Report No: IPP 5/34 May 1990.

2. O.J.W.F. Kardaun, K.S. Riedel, P.J. McCarthy, K. Lackner "A Statistical Approach to Plasma Profile Analysis" Max-Planck Institut fur Plasmaphysik, Report No: IPP 5/35, May 1990.

3. P.J. McCarthy, K.S. Riedel, O.J.W.F. Kardaun, H. Murmann, K. Lackner, Nuclear Fusion, 31, 1595(1991)

4. K.S. Riedel, Smoothing Spline Growth Curves with Covariates C.I.M.S. Report MF-123, March 1992. 
Figure 1
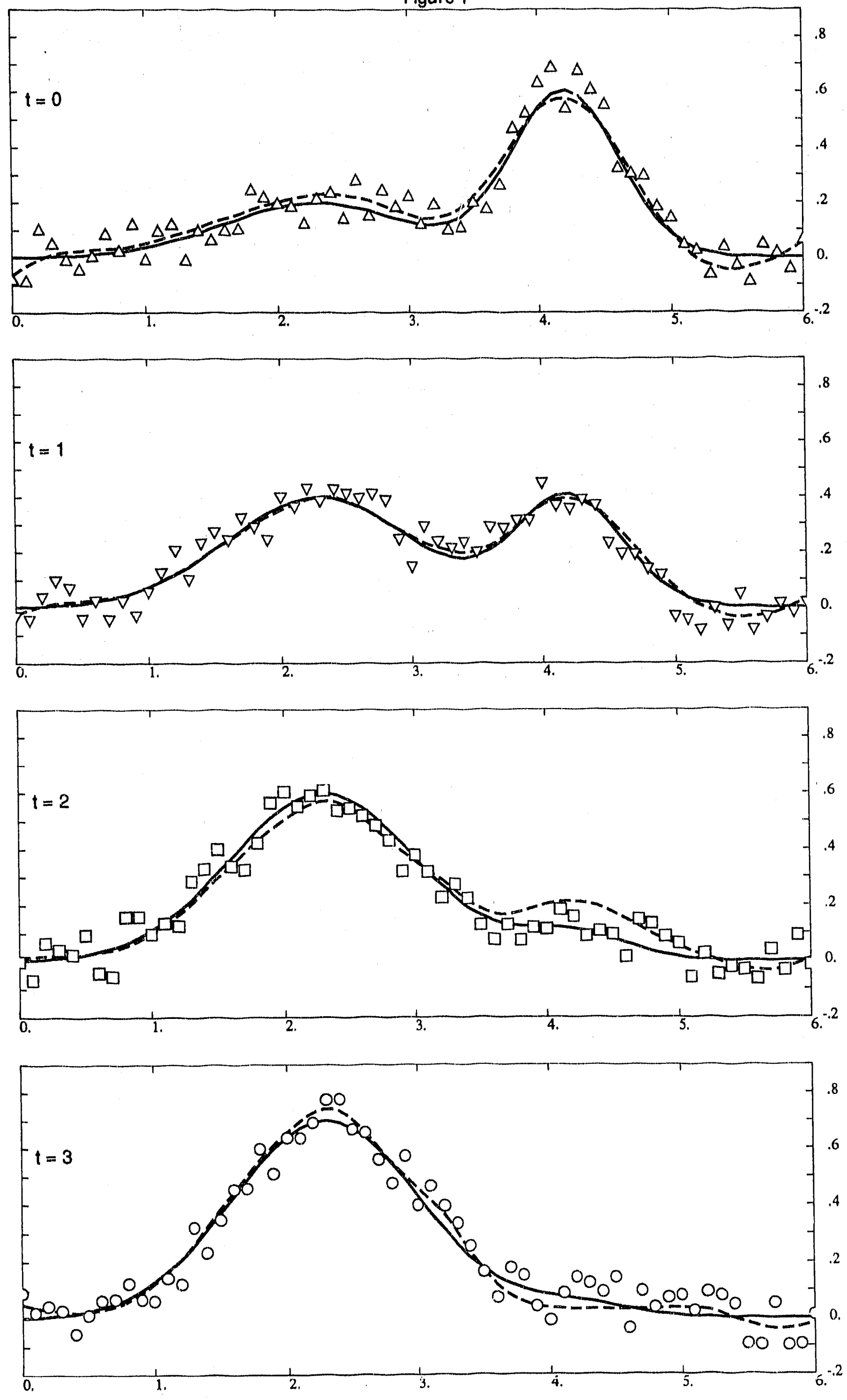
Figure 2

Smoothing of Time Evolution of Noisy Double Gausian

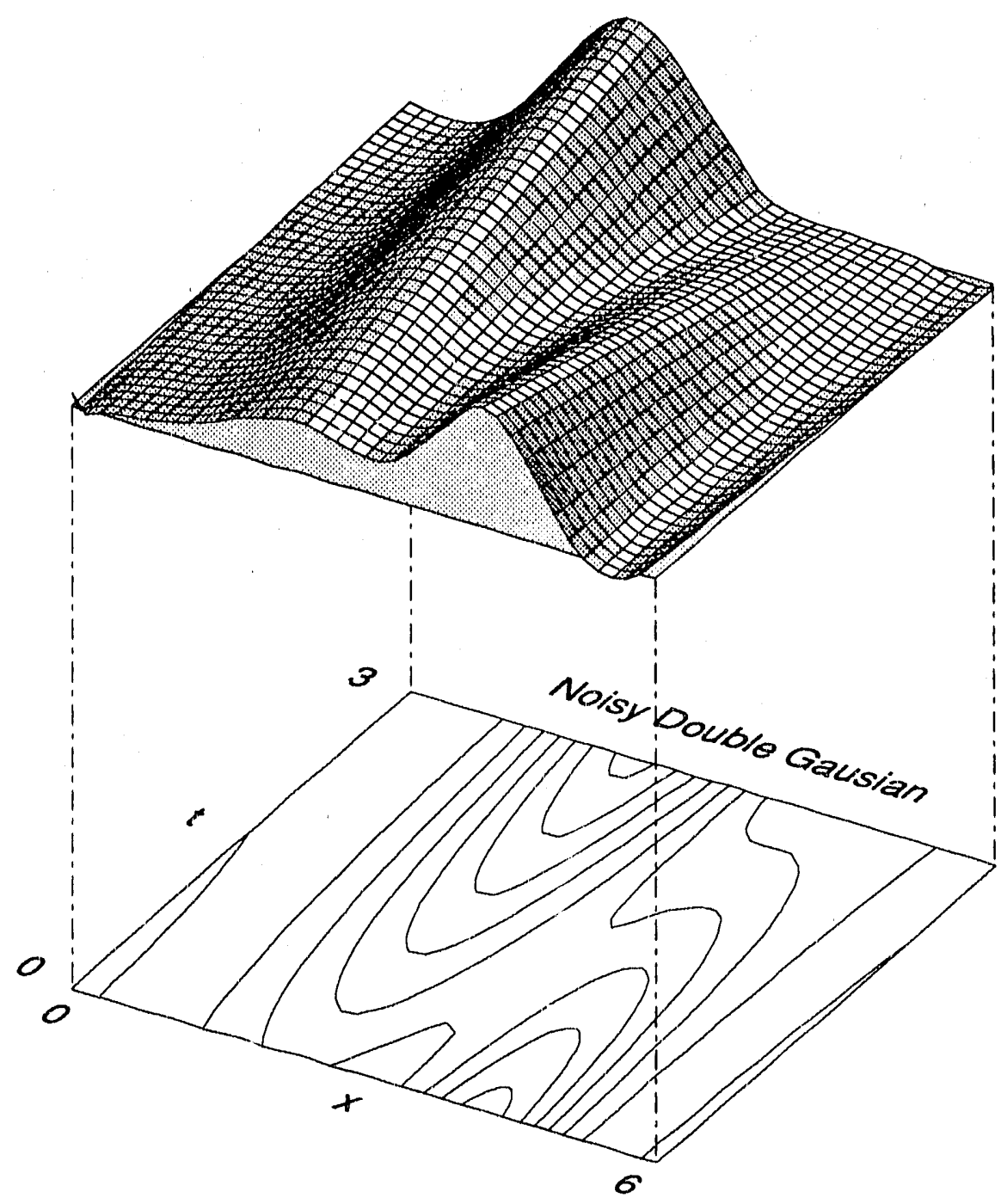



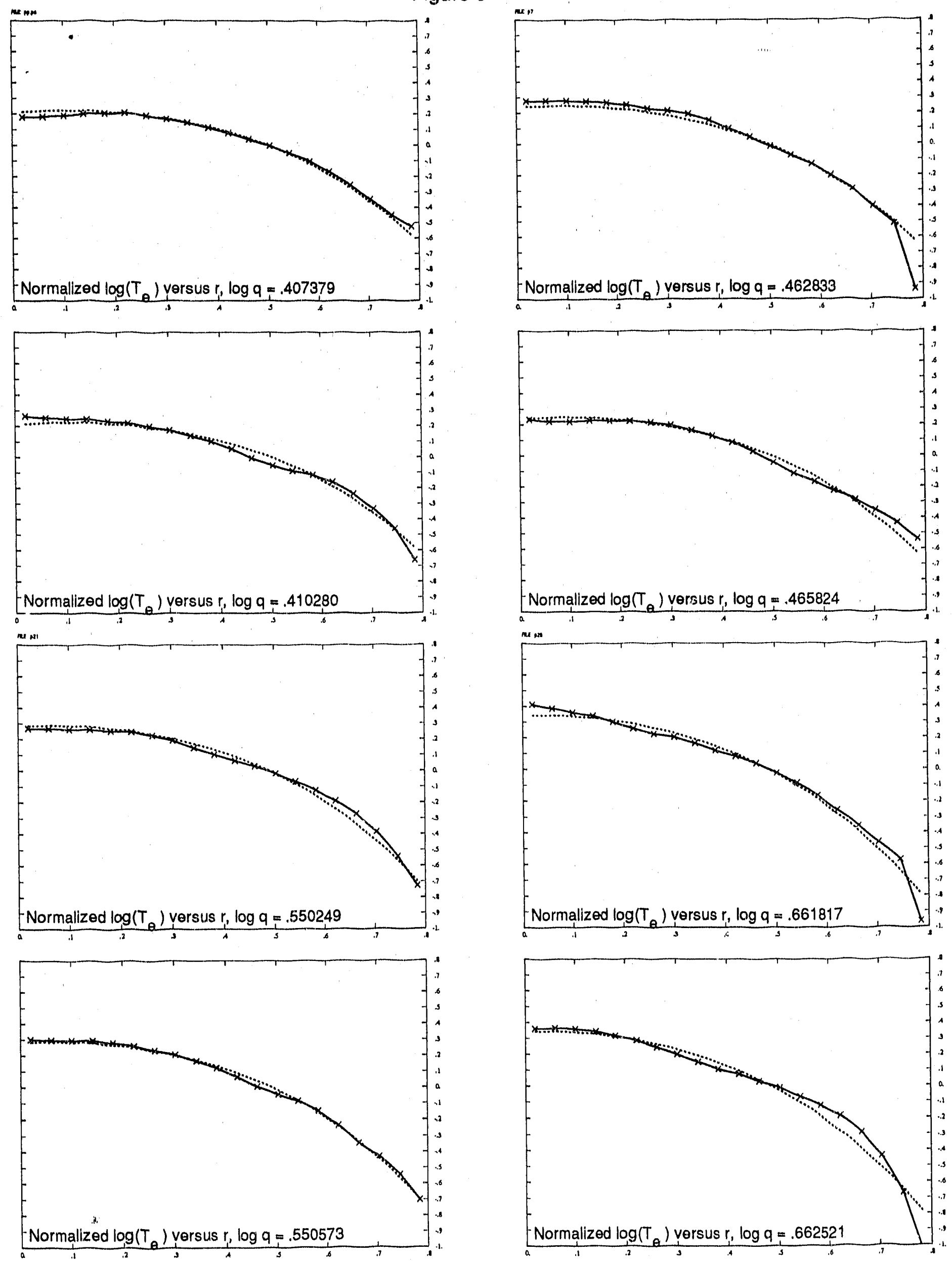
Figure 4

Smoothing of $T_{e}$ obtained from TFTR database

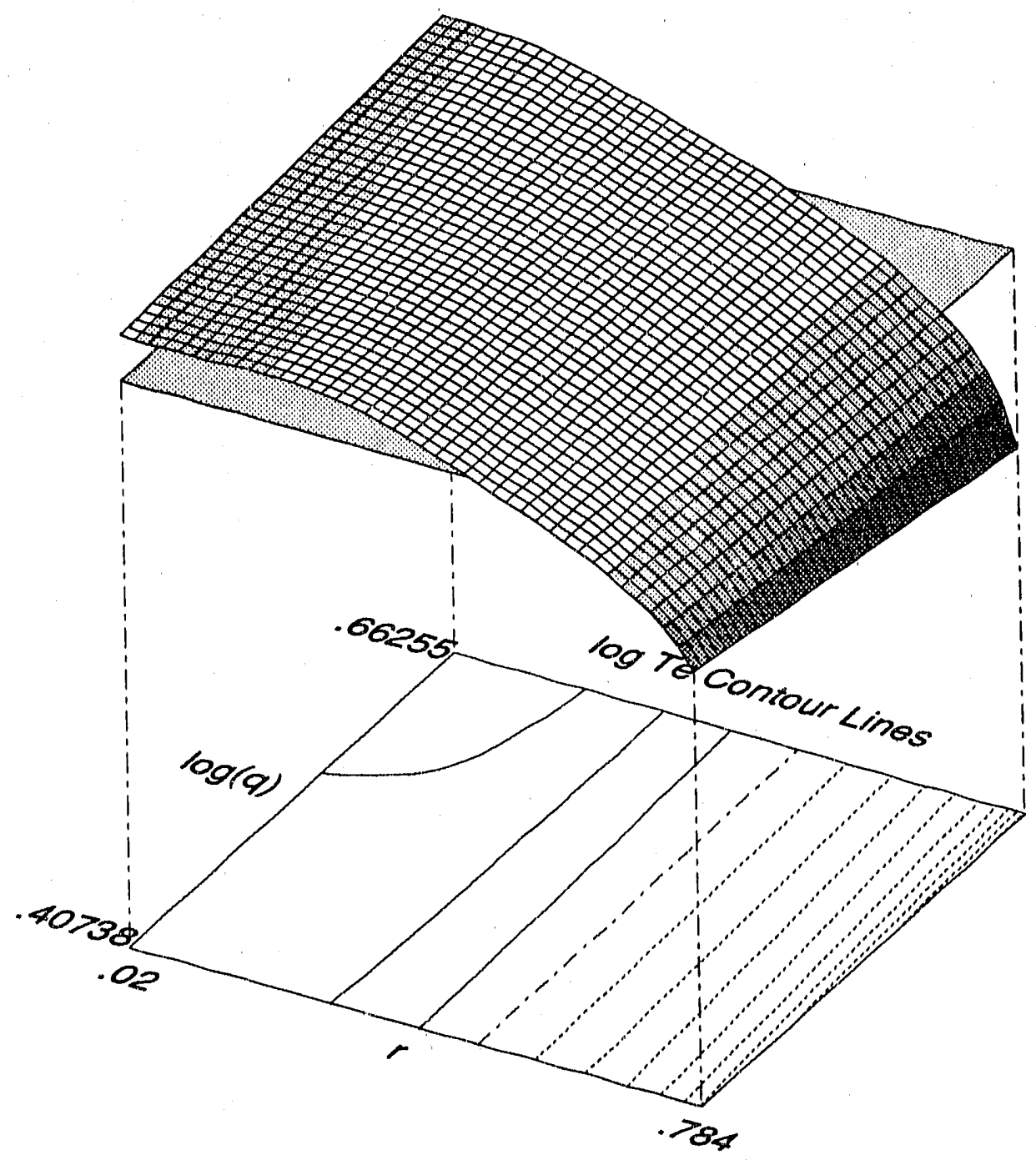




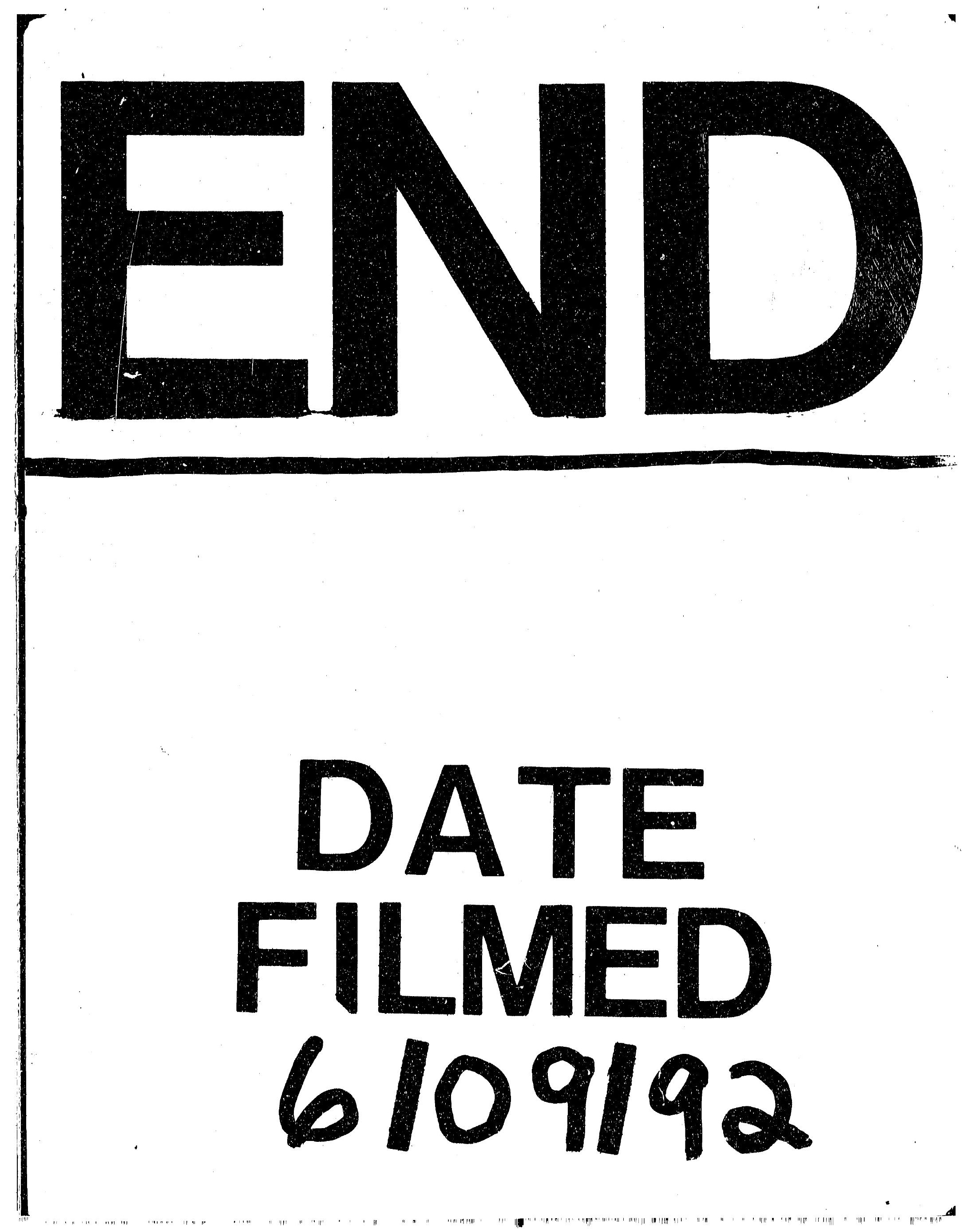


